\title{
Wavefunctions, Green's functions and expectation values in terms of spectral determinants
}

\author{
Martin Sieber ${ }^{1,2} *$ \\ ${ }^{1}$ School of Mathematics, University of Bristol, Bristol BS8 1TW, UK \\ Centro Brasileiro de Pesquisas Físicas, Rua Dr. Xavier Sigaud 150, 22290-180, Rio de Janeiro, Brasil
}

\begin{abstract}
We derive semiclassical approximations for wavefunctions, Green's functions and expectation values for classically chaotic quantum systems. Our method consists of applying singular and regular perturbations to quantum Hamiltonians. The wavefunctions, Green's functions and expectation values of the unperturbed Hamiltonian are expressed in terms of the spectral determinant of the perturbed Hamiltonian. Semiclassical resummation methods for spectral determinants are applied and yield approximations in terms of a finite number of classical trajectories. The final formulas have a simple form. In contrast to Poincaré surface of section methods, the resummation is done in terms of the periods of the trajectories.
\end{abstract}

PACS numbers:

03.65.Sq Semiclassical theories and applications.

05.45.Mt Semiclassical chaos ("quantum chaos").

*E-mail: m.sieber@bristol.ac.uk 


\section{Introduction}

Semiclassical methods have a central role in the study of classically chaotic quantum systems, because they allow to explain properties of quantum systems on the basis of the underlying classical dynamics. A prominent example is the Gutzwiller trace formula that approximates the density of states by a sum over classical periodic orbits. Among its many applications are the explanation of periodic orbit signatures in quantum spectra and the semiclassical evaluation of spectral statistics [1, 2]. For a semiclassical calculation of individual quantum energy levels, however, it is more efficient to use formulations in terms of spectral determinants instead of the density of states. Resummation methods for semiclassical spectral determinants allow to express contributions of long orbits in terms of short ones and yield approximations in terms of a finite number of orbits [3, 4, 5, 6]. As a consequence, a significantly smaller number of periodic orbits is needed. Numerical tests show that these resummed semiclassical sums can be remarkably accurate approximations to quantum spectral determinants [7].

Similar methods for wavefunctions and related quantities have been developed in several articles. Bogomolny derived a semiclassical theory for averages of wavefunctions over small windows of energy and position, starting from the semiclassical Green's function [8]. A corresponding approach in phase space was developed by Berry [9]. One main motivation behind these theories was to explain the scar phenomenon [10]. They apply, however, to averages over several wavefunctions within the considered energy window and do not involve resummations. The first semiclassical formula for single wavefunctions was obtained by Agam and Fishman [11]. Their formula is based on a semiclassical resummation and was used to accurately predict the occurrence of scars in wavefunctions [12]. In subsequent articles Fredholm methods for integral equations on quantum Poincaré surfaces of section were applied to obtain simpler expressions and put the work of Agam and Fishman on a more rigorous basis [13, 14, 15, 16, 17]. In these approaches the resummation is done in terms of the number of intersections of the trajectories with the Poincaré surface instead of their periods. In [18] special properties of tiling billiards on the pseudo-sphere were used to obtain a resummation in terms of the periods for these systems.

In this article we use a different approach. We apply perturbations to a Hamilton in order to express wavefunctions, Green's functions and expectation values in terms of spectral determinants. This allows the application of semiclassical resummation methods for spectral determinants. The resulting formulas are simpler than previous approximations, although they are equivalent to them in the semiclassical limit.

The article is organized as follows. In section 2 we introduce spectral determinants and their semiclassical approximations. In section 3 we discuss singular rank one and rank two perturbations of Hamiltonians which are applied in section 4 to obtain resummed semiclassical approximations for wavefunctions and Green's functions. Corresponding results for expectation values are obtained in section 5 , and section 6 contains the conclusions.

\section{Spectral determinants}

Spectral determinants are functions whose zeros are given by the eigenvalues $E_{m}$ of a quantum Hamiltonian. They are entire functions of the energy $E$, real valued for real $E$, and can be 
expressed as a product over the quantum energy levels $E_{m}$

$$
\Delta(E)=\prod_{m=1}^{\infty} A\left(E, E_{m}\right)\left(E-E_{m}\right)
$$

The coefficients $A\left(E, E_{m}\right)$ are non-vanishing functions of $E$ and make the product convergent. The specific form of these coefficients depends on the chosen regularization. Two common forms of regularizing spectral determinants, the Weierstrass and the zeta regularization, are discussed in appendix $\mathrm{A}$.

As is also discussed in this appendix, the semiclassical approximation to the spectral determinant can be obtained from the (regularized) trace of the Green's function and is expressed in terms of the trajectories of the corresponding classical system [19]

$$
\Delta(E) \approx B(E) \mathrm{e}^{-\mathrm{i} \pi \bar{N}(E)} \zeta(E) .
$$

Here $B(E)$ is a function which is real for real $E$, see appendix A, It will drop out of the final formulas of this article. $\bar{N}(E)$ is the mean spectral counting function which originates from zero-length trajectories. Its leading order asymptotic term is given by the Thomas-Fermi or Weyl estimate

$$
\bar{N}(E) \sim(2 \pi \hbar)^{-D} \int \mathrm{d}^{D} q \mathrm{~d}^{D} p \theta(E-H(\boldsymbol{q}, \boldsymbol{p})) \quad \text { as } \quad \hbar \rightarrow 0
$$

where $\theta(E)$ denotes the Heaviside theta function and $D$ is the dimension.

The function $\zeta(E)$ receives contributions from the classical periodic orbits. In the case of chaotic systems it can be expressed as an Euler product over the periodic orbits. Alternatively, the product can be expanded and expressed as a sum over composite (or pseudo) orbits. We discuss the second form in the following. To each composite orbit, labeled by $n$, corresponds a finite set of primitive periodic orbits $\mathcal{P}_{n}$, and the action of the composite orbit is a linear combination of the actions of the periodic orbits $\mathcal{S}_{n}=\sum_{p \in \mathcal{P}_{n}} m_{p} S_{p}$. The coefficients $m_{p}$ are positive integers and depend on $n$ though we do not indicate this in the notation. More precisely, the index $n$ labels all possible finite linear combinations of actions of primitive periodic orbits with positive coefficients. In addition there is a zero-length contribution, $n=0$, for which $\mathcal{S}_{0}=0$. The zeta function is a sum over all composite orbits in the form

$$
\zeta(E)=\sum_{n=0}^{\infty} C_{n} \exp \left(\frac{\mathrm{i}}{\hbar} \mathcal{S}_{n}\right)
$$

where

$$
C_{n}=\prod_{p \in \mathcal{P}_{n}} \frac{(-1)^{m_{p}} \sigma_{p}^{m_{p}\left(m_{p}-1\right) / 2} \exp \left(-i \frac{\pi}{2} m_{p} \mu_{p}-\frac{m_{p}\left(m_{p}-1\right)}{4} u_{p}\right)}{\left|\prod_{l=1}^{m_{p}} \operatorname{det}\left(M_{p}^{l}-1\right)\right|^{1 / 2}}, \quad n \neq 0,
$$

and $C_{0}=1$. Here $\mu_{p}$ is the Maslov index of the primitive periodic orbit $p$, and $M_{p}$ is its stability matrix. $M_{p}$ has eigenvalues $\sigma_{p} \mathrm{e}^{u_{p}}$ where $\sigma_{p}$ is the sign of the eigenvalues. In (44) with (5) it is assumed that all periodic orbits are unstable. We note that in one-dimensional systems the corresponding approximation for $\zeta(E)$ consists of a finite number of terms, see [19].

Due to the exponential proliferation of periodic orbits in chaotic systems, the sum in (4) is not absolutely convergent for real $E$. Motivated by the Riemann-Siegel formula for the 
Riemann zeta function, Berry and Keating derived a resummation of the sum based on a functional equation of the spectral determinant in $\hbar, \Delta(E, \hbar)=\Delta(E,-\hbar)$ [4]. (A different approach is based on the fact that $\Delta(E)$ is real if $E$ is real [20].) The derivation uses Cauchy integral techniques and the semiclassical sum (44) in regions of the complex $\hbar$-plane where it is absolutely convergent. This resummation makes the sum effectively finite by expressing the contribution of longer composite orbits in terms of shorter ones. The approximation has the form (in the semiclassical regime)

$$
\Delta(E) \approx B(E) 2 \operatorname{Re}^{-\mathrm{i} \pi \bar{N}(E)} \sum_{n=0}^{\infty} C_{n} \exp \left(\frac{\mathrm{i}}{\hbar} \mathcal{S}_{n}\right) \theta^{\operatorname{tr}}\left(T_{H} / 2-\mathcal{T}_{n}\right),
$$

where $\mathcal{T}_{n}=\mathrm{d} \mathcal{S}_{n} / \mathrm{d} E$ are the periods of the composite orbits. $\theta^{\operatorname{tr}}(T)$ is a function which smoothly truncates the sum. It goes from zero to one in a region with width of order $\hbar^{1-D / 2}$ around $T=0$ (for the optimal choice of one free parameter). $T_{H}$ is the Heisenberg time $T_{H}=2 \pi \hbar \bar{d}(E) \propto$ $\hbar^{1-D}$, where $\bar{d}(E)=\bar{N}^{\prime}(E)$ is the mean density of states. In [4] the truncation function is given by a series of terms whose leading term contains a complementary error function, and the correction terms are given by higher order derivatives of the complementary error function and are different from zero only in the vicinity of $T=0$.

Resummed spectral determinants form the basis for the semiclassical approximations in this article. The arguments of Berry and Keating apply also to the more general expansions of spectral determinants that are considered in this article. This is because the spectral determinants satisfy the same functional equation, and the semiclassical expansions have the same general structure as in (2) with (4). The difference is that not all of the trajectories that form the composite orbits are periodic.

In the next section we will consider Hamiltonian with singular perturbations. In this case there are additional contributions from classical trajectories which are not periodic. They enter the semiclassical approximation by means of the Green's function $G\left(\boldsymbol{q}, \boldsymbol{q}^{\prime}, E\right)=\left\langle\boldsymbol{q}\left|(E-H)^{-1}\right| \boldsymbol{q}^{\prime}\right\rangle$ whose semiclassical approximation is given by [1]

$$
G\left(\boldsymbol{q}, \boldsymbol{q}^{\prime}, E\right) \approx \sum_{\gamma} A_{\gamma} \mathrm{e}^{\mathrm{i} S_{\gamma} / \hbar}
$$

where

$$
A_{\gamma}=\frac{2 \pi}{(2 \pi \mathrm{i} \hbar)^{(D+1) / 2}} \sqrt{\left|\frac{1}{\dot{q} \dot{q}^{\prime}} \operatorname{det}^{\prime}\left(\frac{\partial^{2} S_{\gamma}}{\partial \boldsymbol{q} \partial \boldsymbol{q}^{\prime}}\right)\right|} \mathrm{e}^{-i \nu_{\gamma} \pi / 2} .
$$

Here $\gamma$ labels all classical trajectories at energy $E$ from $\boldsymbol{q}^{\prime}$ to $\boldsymbol{q} . S_{\gamma}$ is the action and $\nu_{\gamma}$ the number of conjugate points along $\gamma$. The prime at the determinant in (8) indicates that the determinant involves local coordinates perpendicular to the trajectory.

The approximation (7) is not correct in the limit $\boldsymbol{q}^{\prime} \rightarrow \boldsymbol{q}$, because it does not describe correctly the contribution of those trajectories whose length goes to zero in this limit. The correct contribution of these short-length trajectories is given in this limit, for Hamiltonians of the form $H=\boldsymbol{p}^{2} / 2 m+V(\boldsymbol{q})$, by the free Green's function [21, 22]

$$
G^{\text {free }}\left(\boldsymbol{q}, \boldsymbol{q}^{\prime}, E\right)=\frac{m}{2 i \hbar^{2}}\left(\frac{p}{2 \pi \hbar\left|\boldsymbol{q}-\boldsymbol{q}^{\prime}\right|}\right)^{D / 2-1} H_{D / 2-1}^{(1)}\left(p\left|\boldsymbol{q}-\boldsymbol{q}^{\prime}\right| / \hbar\right),
$$

where $H_{D / 2-1}^{(1)}$ denotes a Hankel function of the first kind [23]. The classical momentum $p$ is evaluated at $\left(\boldsymbol{q}+\boldsymbol{q}^{\prime}\right) / 2$. Formula (9) shows the divergence of the Green's function as $\boldsymbol{q}^{\prime} \rightarrow \boldsymbol{q}$ for $D>1$. The divergence is logarithmic for $D=2$ and proportional to $1 /\left|\boldsymbol{q}-\boldsymbol{q}^{\prime}\right|$ for $D=3$. 
From the imaginary part of expression (9) one can obtain the Thomas-Fermi approximation for the local density of states

$$
d(\boldsymbol{q}, E):=\sum_{m=1}^{\infty}|\psi(\boldsymbol{q})|^{2} \delta\left(E-E_{m}\right)=-\left.\frac{1}{\pi} \operatorname{Im} G(\boldsymbol{q}, \boldsymbol{q}, E+\mathrm{i} \varepsilon)\right|_{\varepsilon \rightarrow 0},
$$

in the form

$$
\bar{d}(\boldsymbol{q}, E) \approx \frac{2 m \pi^{D / 2} p^{D-2}}{(2 \pi \hbar)^{D} \Gamma(D / 2)}=(2 \pi \hbar)^{-D} \int \mathrm{d}^{D} p \delta\left(E-\frac{p^{2}}{2 m}-V(\boldsymbol{q})\right) .
$$

Integration over $\boldsymbol{q}$ and $E$ yields (3) for the Hamiltonians considered.

For convenience in the next sections we introduce the following notation. Let $a$ be any quantity which has a semiclassical expansion in terms of classical trajectories or composite

trajectories in the form $a^{\mathrm{sc}}=\sum_{n} a_{n} \mathrm{e}^{\mathrm{i} s_{n} / \hbar}$. Then we denote the smoothly truncated semiclassical sum by

$$
a^{\mathrm{sc}, \operatorname{tr}}=\sum_{n} a_{n} \mathrm{e}^{\mathrm{i} s_{n} / \hbar} \theta^{\operatorname{tr}}\left(T_{H} / 2-t_{n}\right)
$$

where sc stands for semiclassical and tr for truncation. In this notation $\Delta(E) \approx 2 \operatorname{Re} \Delta^{\mathrm{sc}, \operatorname{tr}}(E)$, and the semiclassical energies satisfy the condition

$$
2 \operatorname{Re} \Delta^{\mathrm{sc}, \operatorname{tr}}\left(E_{m}^{\mathrm{sc}}\right)=0 .
$$

\section{Singular perturbations}

We discuss in this section singular rank one and rank two perturbations as a means to derive semiclassical approximations for wavefunctions and Green's functions in the next section. These kind of perturbations can be defined by the applying the method of self-adjoint extension. It is instructive to start with the example of a one-dimensional delta-function potential, for which the correct results can be obtained by simpler calculations.

Consider a quantum Hamiltonian of the form

$$
H=H_{0}+\lambda A,
$$

where $H_{0}$ is the unperturbed Hamiltonian and the perturbation has the form $A=|q\rangle\langle q|$. The eigenstates of $H$ are determined by the condition

$$
0=\left(H_{0}-E\right)|\psi\rangle+\lambda|q\rangle\langle q \mid \psi\rangle .
$$

Multiplying this equation from the left by $\langle q| G_{0}$, where $G_{0}=\left(E-H_{0}\right)^{-1}$ is the resolvent of the unperturbed Hamiltonian $H_{0}$, yields

$$
0=\left(1-\lambda\left\langle q\left|G_{0}\right| q\right\rangle\right)\langle q \mid \psi\rangle .
$$

The solutions are given by unperturbed energies $E_{m}$ if the corresponding eigenstate vanishes at $q$, and by the zeros of the bracket on the right-hand side.

The resolvent of the perturbed Hamiltonian can be obtained from the general relation $G=G_{0}+G_{0} \lambda A G$ from which follows that

$$
G=\frac{1}{1-G_{0} \lambda|q\rangle\langle q|} G_{0} .
$$


The eigenvalues of the Hamiltonian $H$ are given by the poles of the resolvent $G$. Hence the new spectral determinant has the form

$$
\Delta(E)=\Delta_{0}(E) \operatorname{det}\left(1-\lambda G_{0}|q\rangle\langle q|\right) .
$$

Applying $\operatorname{det} M=\mathrm{e}^{\operatorname{Tr} \log M}$ yields

$$
\operatorname{det}\left(1-\lambda G_{0}|q\rangle\langle q|\right)=\mathrm{e}^{-\sum_{n=1}^{\infty} \frac{\lambda^{n}}{n} \operatorname{Tr}\left(G_{0}|q\rangle\langle q|\right)^{n}}=\mathrm{e}^{-\sum_{n=1}^{\infty} \frac{\lambda^{n}}{n}\left(\left\langle q\left|G_{0}\right| q\right\rangle\right)^{n}}=1-\lambda\left\langle q\left|G_{0}\right| q\right\rangle,
$$

and the spectral determinant follows as

$$
\Delta(E)=\Delta_{0}(E)\left[1-\lambda G_{0}(q, q, E)\right]
$$

where $G_{0}\left(q, q^{\prime}, E\right)=\left\langle q\left|G_{0}\right| q^{\prime}\right\rangle$ is the Green's function. Note that the poles of the Green's function cancel the zeros of $\Delta_{0}(E)$ in (20), if they are non-degenerate and the corresponding wavefunction does not vanish at $q$. The zeros of $\Delta(E)$ are given by the zeros of the expression in the bracket, plus the zeros of $\Delta_{0}(E)$ with a degeneracy that is reduced by one (except if all functions in the eigenspace vanish at $q$ ). This agrees with the discussion after equation (16).

An alternative presentation of the Green's function can be obtained by using the relation

$$
\frac{1}{1-G_{0} \lambda|q\rangle\langle q|}=1+\frac{\lambda|q\rangle\langle q| G_{0}}{1-\lambda\left\langle q\left|G_{0}\right| q\right\rangle},
$$

which can be verified by multiplying both sides with the denominators. Inserting this into (17) results in

$$
G(E)=G_{0}+G_{0}|q\rangle \frac{\lambda}{1-\lambda\left\langle q\left|G_{0}\right| q\right\rangle}\langle q| G_{0} .
$$

If the fraction is expanded into a geometric series one obtains an expression that can be interpreted as a sum over diffractive orbits [24].

It is well known that a delta-function potential is not well-defined in two or three dimensions. This is reflected by the fact that $\left\langle\boldsymbol{q}\left|G_{0}\right| \boldsymbol{q}\right\rangle$ is infinite in these cases. The problem of properly defining a pointlike perturbation for $D=2$ and $D=3$ has a long history, and the extensive literature about it is reviewed in [25, 26]. There exist several methods for dealing with this problem. The standard method nowadays is to apply the theory of self-adjoint extension to a Hamiltonian from whose domain one point has been removed, see e.g. [27, 25, 28, 26, 29]. The resolvent of the Hamiltonian that is obtained by the self-adjoint extension is related by Krein's formula to the resolvent of the unperturbed Hamiltonian. The resulting expression for the resolvent of the perturbed Hamiltonian has a very similar form as (22). The difference is that the Green's function $G_{0}(\boldsymbol{q}, \boldsymbol{q}, E)=\left\langle\boldsymbol{q}\left|G_{0}\right| \boldsymbol{q}\right\rangle$ is replaced by a regularized expression.

$$
G(E)=G_{0}+G_{0}|\boldsymbol{q}\rangle \frac{\lambda}{1-\lambda G_{0}^{\mathrm{reg}}(\boldsymbol{q}, \boldsymbol{q}, E)}\langle\boldsymbol{q}| G_{0},
$$

and the corresponding expression for the determinant is

$$
\Delta_{1}(E, \lambda, \boldsymbol{q})=\Delta_{0}(E)\left[1-\lambda G_{0}^{\mathrm{reg}}(\boldsymbol{q}, \boldsymbol{q}, E)\right] .
$$

The regularization of the Green's function is not unique. Different regularizations can differ by an arbitrary real constant [29]. However, all different regularizations lead to the same family of self-adjoint extensions of the Hamiltonian. This can be seen from the fact that a change 
of the regularized Green's function in (23) by an additive constant $c$ can be compensated by redefining the perturbation parameter according to $\lambda \rightarrow \lambda /(1+c \lambda)$.

We will regularize the Green's function in the following by subtracting its divergent part in the limit $\boldsymbol{q}^{\prime} \rightarrow \boldsymbol{q}$. For Hamiltonians of the form $H_{0}=\boldsymbol{p}^{2} / 2 m+V(\boldsymbol{q})$ the asymptotic behavior of the Green's function in the limit $\boldsymbol{q}^{\prime} \rightarrow \boldsymbol{q}$ is given by the free Green's function (9), and we define

$$
\begin{array}{ll}
\left.G^{\mathrm{reg}}(\boldsymbol{q}, \boldsymbol{q}, E)=\lim _{\boldsymbol{q}^{\prime} \rightarrow \boldsymbol{q}}\left[G_{(}, \boldsymbol{q}, \boldsymbol{q}^{\prime}, E\right)-\frac{m}{\pi \hbar^{2}} \log \left(k_{0}\left|\boldsymbol{q}-\boldsymbol{q}^{\prime}\right|\right)\right] & (D=2), \\
\left.G^{\mathrm{reg}}(\boldsymbol{q}, \boldsymbol{q}, E)=\lim _{\boldsymbol{q}^{\prime} \rightarrow \boldsymbol{q}}\left[G_{(} \boldsymbol{q}, \boldsymbol{q}^{\prime}, E\right)+\frac{m}{2 \pi \hbar^{2}\left|\boldsymbol{q}-\boldsymbol{q}^{\prime}\right|}\right] & (D=3) .
\end{array}
$$

The constant $k_{0}$ in the first line of (25) is arbitrary and is included for dimensional reasons. It follows from the freedom in the choice of the regularization that the constant $\lambda$ cannot, in general, be interpreted as the physical strength of the scatterer. It is interesting to note that a possible physical interpretation can be obtained by introducing a new parameter $a$

$$
\frac{1}{\lambda}=-\frac{m}{\pi \hbar^{2}} \log \left(k_{0} a\right) \quad(D=2), \quad \frac{1}{\lambda}=\frac{m}{2 \pi \hbar^{2} a} \quad(D=3) .
$$

Then formula (23) is valid for the scattering from a small but finite scatterer of radius $a$ in the limit that $a$ is much smaller than the wavelength [30].

The semiclassical approximation for the determinant (24) is obtained by applying the semiclassical resummation that was discussed in the last section. It results in

$$
\Delta_{1}(E, \lambda, \boldsymbol{q}) \approx 2 \operatorname{Re}\left[\Delta_{0}(E)\left(1-\lambda G_{0}^{\mathrm{reg}}(\boldsymbol{q}, \boldsymbol{q}, E)\right)\right]^{\mathrm{sc}, \mathrm{tr}} .
$$

This has the following interpretation. $\Delta_{0}(E)$ and $\left(1-\lambda G_{0}^{\mathrm{reg}}(\boldsymbol{q}, \boldsymbol{q}, E)\right)$ both have a semiclassical expansion in terms of a sum over classical trajectories. These two sums are multiplied, and one obtains a sum over combined trajectories with actions $\mathcal{S}_{n}+S_{\gamma}$. These combined trajectories might be viewed as a new form of composite orbits. The corresponding times along the orbits are $\mathcal{T}_{n}+T_{\gamma}$. The sum over the combined trajectories is then smoothly truncated at half the Heisenberg time.

In the following we will consider also rank two perturbations which correspond to selfadjoint extensions of a Hamiltonian from whose domain two points are removed [31, 32, 26]. They formally correspond to a perturbation by an operator of the form

$$
" \lambda_{1}\left|\boldsymbol{q}_{1}\right\rangle\left\langle\boldsymbol{q}_{1}|+\mu| \boldsymbol{q}_{1}\right\rangle\left\langle\boldsymbol{q}_{2}|+\bar{\mu}| \boldsymbol{q}_{2}\right\rangle\left\langle\boldsymbol{q}_{1}\left|+\lambda_{2}\right| \boldsymbol{q}_{2}\right\rangle\left\langle\boldsymbol{q}_{2}\right| "
$$

where $\lambda_{1}$ and $\lambda_{2}$ are real and $\mu=\mu_{r}+\mathrm{i} \mu_{i}$ is complex. In this case the determinant takes the form

$$
\Delta(E)=\Delta_{0}(E) \operatorname{det} F,
$$

where $F$ is the following $2 \times 2$ matrix

$$
F=1-\left(\begin{array}{cc}
G_{0}^{\mathrm{reg}}\left(\boldsymbol{q}_{1}, \boldsymbol{q}_{1}, E\right) & G_{0}\left(\boldsymbol{q}_{1}, \boldsymbol{q}_{2}, E\right) \\
G_{0}\left(\boldsymbol{q}_{2}, \boldsymbol{q}_{1}, E\right) & G_{0}^{\mathrm{reg}}\left(\boldsymbol{q}_{2}, \boldsymbol{q}_{2}, E\right)
\end{array}\right)\left(\begin{array}{cc}
\lambda_{1} & \mu \\
\bar{\mu} & \lambda_{2}
\end{array}\right) .
$$

We will apply the determinant (28) in the following for $\lambda_{1}=\lambda_{2}=0$ and denote it by

$$
\Delta_{2}\left(E, \mu, \boldsymbol{q}_{1}, \boldsymbol{q}_{2}\right)=\Delta_{0}(E) f\left(E, \mu, \boldsymbol{q}_{1}, \boldsymbol{q}_{2}\right) \text {, }
$$


where the function $f$ follows as

$$
\begin{aligned}
f\left(E, \mu, \boldsymbol{q}_{1}, \boldsymbol{q}_{2}\right)= & 1-\mu G_{0}\left(\boldsymbol{q}_{2}, \boldsymbol{q}_{1}, E\right)-\bar{\mu} G_{0}\left(\boldsymbol{q}_{1}, \boldsymbol{q}_{2}, E\right) \\
& +|\mu|^{2}\left[G_{0}\left(\boldsymbol{q}_{1}, \boldsymbol{q}_{2}, E\right) G_{0}\left(\boldsymbol{q}_{2}, \boldsymbol{q}_{1}, E\right)-G_{0}^{\mathrm{reg}}\left(\boldsymbol{q}_{1}, \boldsymbol{q}_{1}, E\right) G_{0}^{\mathrm{reg}}\left(\boldsymbol{q}_{2}, \boldsymbol{q}_{2}, E\right)\right] .
\end{aligned}
$$

The determinants $\Delta_{2}$ and $\Delta_{1}$ are entire functions of $E$, because the poles that arise from the Green's functions are cancelled by the zeros of $\Delta_{0}$. The corresponding semiclassical approximation for $\Delta_{2}$ is obtained by inserting the semiclassical expansion for $\Delta_{0}$ and applying the resummation

$$
\Delta_{2}\left(E, \mu, \boldsymbol{q}_{1}, \boldsymbol{q}_{2}\right) \approx 2 \operatorname{Re}\left[\Delta_{0}(E) f\left(E, \mu, \boldsymbol{q}_{1}, \boldsymbol{q}_{2}\right)\right]^{\mathrm{sc}, \mathrm{tr}},
$$

which is to interpreted according to the discussion after equation (27).

\section{Wavefunctions and Green's functions}

The effect that a perturbation by a pointlike scatterer has on an energy level depends on the value of the wavefunction at this point. The semiclassical approach in this section is based on this relation. Consider one non-degenerate eigenvalue $E_{m}$ of the unperturbed Hamiltonian $H_{0}$. The regularized Green's function in the eigenvalue equation (24) has a pole at $E=E_{m}$ with residue $\left|\psi_{m}(\boldsymbol{q})\right|^{2}$. Hence we find that the determinant of the perturbed problem, evaluated at the unperturbed energy $E=E_{m}$, satisfies

$$
\Delta_{1}\left(E_{m}, \lambda, \boldsymbol{q}\right)=-\lambda \lim _{E \rightarrow E_{m}} \Delta_{0}(E) \frac{\left|\psi_{m}(\boldsymbol{q})\right|^{2}}{E-E_{m}}=-\lambda \Delta_{0}^{\prime}\left(E_{m}\right)\left|\psi_{m}(\boldsymbol{q})\right|^{2} .
$$

We take a derivative with respect to $\lambda$ on both sides of this equation. This leads to an expression for the absolute square of the wavefunction as a quotient of derivatives of the spectral determinant

$$
\left|\psi_{m}(\boldsymbol{q})\right|^{2}=-\left.\frac{\frac{\partial}{\partial \lambda} \Delta_{1}(E, \lambda, \boldsymbol{q})}{\frac{\partial}{\partial E} \Delta_{1}(E, \lambda, \boldsymbol{q})}\right|_{\substack{\lambda=0 \\ E=E_{m}}}
$$

Inserting the semiclassical approximation (27) results in

$$
\left|\psi_{m}(\boldsymbol{q})\right|^{2} \approx \frac{\operatorname{Re}\left[\Delta_{0}\left(E_{m}\right) G_{0}\left(\boldsymbol{q}, \boldsymbol{q}, E_{m}\right)\right]^{\mathrm{sc}, \mathrm{tr}}}{\operatorname{Re}\left[\Delta_{0}^{\prime}\left(E_{m}\right)\right]^{\mathrm{sc}, \mathrm{tr}}}
$$

This is the result for the semiclassical approximation for the modulus square of the wavefunction. It gives an approximation in terms of a double sum over composite periodic orbits and closed orbits from $\boldsymbol{q}$ to $\boldsymbol{q}$ which is cut off smoothly when the sum of the times along the orbits is half the Heisenberg time. The energies $E_{m}$ in (35) are determined semiclassically by (13)).

Let us discuss the difference to previous semiclassical formulas for the square modulus of the wavefunction. Agam and Fishman obtain their approximation by assuming that only closed orbits which are almost periodic are important [11. They describe these closed orbits in terms of nearby periodic orbits and perform a sum over all repetitions of the periodic orbits before applying a resummation to the semiclassical sums. Auslaender and Fishman [18] use the fact that for tiling billiards on the pseudosphere there exists an exact relation between closed orbits and periodic orbits. They do not assume that closed orbits are almost periodic, but perform also the sum over repetitions. Equation (35) is simpler than the formulas in these articles 
mainly because of this difference in the resummation. Furthermore, it is not assumed that the contributing closed orbits are almost periodic. Other approximations for the square modulus of the wavefunction were derived by Poincaré surface of section methods [14, 33, 15, 16]. In these cases the resummation is based on the semiclassical unitarity of the transfer operator, or the scattering matrix, and is done in terms of the number of intersections with the Poincaré surface.

We continue with a further discussion of (35). One point to note is that (35) is independent of the choice of the regularization of the Green's function. This is because the real part of the zero-length contribution to the Green's function is multiplied in (35) by $\operatorname{Re}\left[\Delta_{0}\left(E_{m}\right)\right]^{\text {sc,tr }}$ which vanishes.

There is, however, a non-vanishing contribution to (35) from the imaginary part of the zero-length contributions to the Green's function. Using (10) we find that this contribution is given by

$$
\frac{\operatorname{Im}\left[\Delta_{0}\left(E_{m}\right)\right]^{\mathrm{sc}, \operatorname{tr}}}{\operatorname{Re}\left[\Delta_{0}^{\prime}\left(E_{m}\right)\right]^{\mathrm{sc}, \operatorname{tr}}} \pi \bar{d}\left(\boldsymbol{q}, E_{m}\right) .
$$

The derivative of the semiclassical determinant, equation (2) with (3) and (44) consists semiclassically of two terms (the derivative of $B(E)$ is multiplied by a vanishing term)

$$
\operatorname{Re}\left[\Delta_{0}^{\prime}\left(E_{m}\right)\right]^{\mathrm{sc}, \operatorname{tr}}=\pi \bar{d}\left(E_{m}\right) \operatorname{Im}\left[\Delta_{0}\left(E_{m}\right)\right]^{\mathrm{sc}, \operatorname{tr}}-\frac{1}{\hbar} B\left(E_{m}\right) \operatorname{Im}\left[\mathrm{e}^{-\mathrm{i} \pi \bar{N}\left(E_{m}\right)} \sum_{n=1}^{\infty} T_{n} C_{n} \exp \left(\frac{\mathrm{i}}{\hbar} \mathcal{S}_{n}\right)\right]^{\mathrm{tr}}
$$

Note that $\bar{d}(E) \propto \hbar^{-D}$. If we take only the first term into account we obtain the following approximation

$$
\left|\psi_{m}(\boldsymbol{q})\right|^{2} \approx \frac{\bar{d}\left(\boldsymbol{q}, E_{m}\right)}{\bar{d}\left(E_{m}\right)}
$$

This corresponds to a projection of the classical microcanonical distribution onto the coordinate space. When integrated over some small area, this is consistent with the quantum ergodicity theorem which, roughly speaking, states that almost all matrix elements of smooth operators tend to the microcanonical average in the semiclassical limit [34, 35, 36].

Equation (35) can applied for an examination of scars. If a small smoothing of width $\Delta q$ is applied, then the initial and final momenta of the contributing closed orbits differ at most by a value of the order of $\hbar / \Delta q$, and they can be described in terms of nearby periodic orbits. Since the $q$-dependence in (35) is in terms of the semiclassical Green's function, Bogomolny's analysis of the structure of wavefunctions near periodic orbits can be applied without additional smoothing over an energy window. An effect of the resummation is that the contribution of a trajectory $\gamma$ is weighted by the semiclassical expression for the spectral determinant, truncated at $T_{H} / 2-T_{\gamma}$. The semiclassical criterion for scars of Agam and Fishman [12, 18] can be obtained by integrating (35) over some tube around the periodic orbits.

Equation (35) does not contain any information about the phase of the wavefunction. It is possible to obtain this information by a similar approach. For this purpose we consider the determinant (30) for the rank two perturbation. A calculation similar to (33) leads to

$$
\psi_{m}(\boldsymbol{q}) \overline{\psi_{m}\left(\boldsymbol{q}^{\prime}\right)}=-\left.\frac{\left[\frac{\partial}{\partial \mu_{r}}+\mathrm{i} \frac{\partial}{\partial \mu_{i}}\right] \Delta_{2}\left(E, \mu, \boldsymbol{q}, \boldsymbol{q}^{\prime}\right)}{2 \frac{\partial}{\partial E} \Delta_{2}\left(E, \mu, \boldsymbol{q}, \boldsymbol{q}^{\prime}\right)}\right|_{\substack{\mu=0 \\ E=E_{m}}}
$$


We insert the semiclassical approximation (32) and obtain

$$
\psi_{m}(\boldsymbol{q}) \overline{\psi_{m}\left(\boldsymbol{q}^{\prime}\right)} \approx \frac{\left[\Delta_{0}\left(E_{m}\right) G_{0}\left(\boldsymbol{q}, \boldsymbol{q}^{\prime}, E_{m}\right)+\overline{\Delta_{0}\left(E_{m}\right) G_{0}\left(\boldsymbol{q}^{\prime}, \boldsymbol{q}, E_{m}\right)}\right]^{\mathrm{sc}, \mathrm{tr}}}{2 \operatorname{Re}\left[\Delta_{0}^{\prime}\left(E_{m}\right)\right]^{\mathrm{sc}, \mathrm{tr}}} .
$$

This is a double sum over composite orbits and trajectories from $\boldsymbol{q}^{\prime}$ to $\boldsymbol{q}$, respectively from $\boldsymbol{q}$ to $\boldsymbol{q}^{\prime}$, which is truncated smoothly when the sum of the times along orbits and trajectories is half the Heisenberg time. Semiclassical approximations for this quantity have been obtained before by Poincaré surface of section methods, see [15]. (40) together with (35) allows to calculate the normalized wavefunction semiclassically everywhere.

In the case of an degenerate energy level $E_{m}$ equations (35) and (40) are formulas for $\left\langle\boldsymbol{q}\left|P_{m}\right| \boldsymbol{q}\right\rangle$ and $\left\langle\boldsymbol{q}\left|P_{m}\right| \boldsymbol{q}^{\prime}\right\rangle$, respectively, where $P_{m}$ is the projection operator onto the corresponding eigenspace. If the level is $d$-fold degenerate one can choose a basis of the eigenspace for which $d-1$ basis states vanish at $\boldsymbol{q}$. Hence equations (35) and (40) are formulas for the remaining state which, in general, does not vanish at $\boldsymbol{q}$.

The Green's function can be obtained in a very similar way. From (30) follows that

$$
\Delta_{0}(E) G_{0}\left(\boldsymbol{q}, \boldsymbol{q}^{\prime}, E\right)=\left.\frac{1}{2}\left[\frac{\partial}{\partial \mu_{r}}+\mathrm{i} \frac{\partial}{\partial \mu_{i}}\right] \Delta_{2}\left(E, \mu, \boldsymbol{q}, \boldsymbol{q}^{\prime}\right)\right|_{\mu=0} .
$$

We insert the semiclassical approximation for the determinant (32) and obtain

$$
G_{0}\left(\boldsymbol{q}, \boldsymbol{q}^{\prime}, E\right) \approx \frac{\left[\Delta_{0}(E) G_{0}\left(E, \boldsymbol{q}, \boldsymbol{q}^{\prime}\right)+\overline{\Delta_{0}(E) G_{0}\left(E, \boldsymbol{q}^{\prime}, \boldsymbol{q}\right)}\right]^{\mathrm{sc}, \mathrm{tr}}}{2 \operatorname{Re}\left[\Delta_{0}(E)\right]^{\mathrm{sc}, \mathrm{tr}}} .
$$

As in (40) this is a double sum over composite orbits and open trajectories. Resummed expressions for the Green's function have been obtained by Poincaré section methods in [13, 14, 15], and for $G_{0}^{\text {reg }}(\boldsymbol{q}, \boldsymbol{q}, E)$ for tiling billiards on the pseudosphere in [18]. In appendix B] we discuss an example of a one-dimensional system for which formula (42), with a sharp cut-off, yields the exact Green's function.

Given the approximation (42) it is straightforward to obtain resummed formulas for other quantities that are related to the Green's function. For example, the Wigner transform of the Green function is

$$
W_{0}(\boldsymbol{x}, E)=\int \mathrm{d}^{D} q^{\prime} \mathrm{e}^{-\mathrm{i} \boldsymbol{p} \boldsymbol{q}^{\prime} / \hbar} G_{0}\left(\boldsymbol{q}+\frac{1}{2} \boldsymbol{q}^{\prime}, \boldsymbol{q}-\frac{1}{2} \boldsymbol{q}^{\prime}, E\right)=h^{D} \sum_{m} \frac{W_{m}(\boldsymbol{x})}{E-E_{m}},
$$

where $\boldsymbol{x}=(\boldsymbol{q}, \boldsymbol{p})$ and $W_{m}(\boldsymbol{x})$ is the Wigner function of the $m$-th eigenstate with energy $E_{m}$. Using (42) and performing the integrals in (43) in stationary phase approximation results in

$$
W_{0}(\boldsymbol{x}, E) \approx \frac{\left[\Delta_{0}(E) W_{0}(\boldsymbol{x}, E)+\overline{\Delta_{0}(E) W_{0}(\boldsymbol{x}, E)}\right]^{\mathrm{sc}, \mathrm{tr}}}{2 \operatorname{Re}\left[\Delta_{0}(E)\right]^{\mathrm{sc}, \mathrm{tr}}},
$$

where the semiclassical approximation to the Wigner function $W_{0}$ is given by classical trajectories that satisfy the midpoint rule $\boldsymbol{x}=\left(\boldsymbol{x}_{f}+\boldsymbol{x}_{i}\right) / 2$ where $\boldsymbol{x}_{f}$ and $\boldsymbol{x}_{i}$ are the final and initial points in phase space [9, 37]. The action terms in (44) are of the form $\mathcal{S}_{n}+S_{\gamma}-\boldsymbol{q}^{\prime} \boldsymbol{p}$, where $\boldsymbol{q}^{\prime}$ is determined by the stationary phase condition, but the resummation is done in terms of $\mathcal{T}_{n}+T_{\gamma}$. One may speculate that (44) holds also for a resummation in terms of $\frac{\mathrm{d}}{\mathrm{d} E}\left[\mathcal{S}_{n}+S_{\gamma}-\boldsymbol{q}^{\prime} \boldsymbol{p}\right]$, and that this can be derived, for example, by starting with a different perturbation.

The corresponding approximation for the Wigner functions of the eigenstates is given by

$$
W_{m}(\boldsymbol{x}) \approx \frac{\left[\Delta_{0}\left(E_{m}\right) W_{0}\left(\boldsymbol{x}, E_{m}\right)+\overline{\Delta_{0}\left(E_{m}\right) W_{0}\left(\boldsymbol{x}, E_{m}\right)}\right]^{\mathrm{sc}, \mathrm{tr}}}{2 h^{D} \operatorname{Re}\left[\Delta_{0}^{\prime}\left(E_{m}\right)\right]^{\mathrm{sc}, \mathrm{tr}}} .
$$




\section{$5 \quad$ Expectation values}

In this section we apply a similar approach to expectation values. Consider the perturbation of a Hamiltonian by a self-adjoint operator $A$ in the form $H=H_{0}+\lambda A$, and let $\Delta(E, \lambda)$ be the associated spectral determinant. As before we denote the determinant of the unperturbed Hamiltonian by $\Delta_{0}(E)$. In contrast to the previous sections we assume now that $A$ is smooth so that the spectral determinant is semiclassically given only in terms of periodic orbits (and not diffractive orbits). Then, if $E_{m}(\lambda)$ is a non-degenerate energy level, and $E_{m}$ denotes $E_{m}(0)$, it follows from the form (11) that

$$
\left.\frac{\partial}{\partial \lambda} \Delta(E, \lambda)\right|_{\substack{\lambda=0 \\ E=E_{m}}}=-\left.\left.\frac{\partial E_{m}(\lambda)}{\partial \lambda}\right|_{\lambda=0} \frac{\Delta_{0}(E)}{E-E_{m}}\right|_{E \rightarrow E_{m}}=-\left\langle\psi_{m}|A| \psi_{m}\right\rangle \Delta_{0}^{\prime}\left(E_{m}\right)
$$

where the second equality follows from first order perturbation theory. Hence the expectation value of the operator $A$ in the state $\left|\psi_{m}\right\rangle$ can be written as a quotient of two partial derivatives of the spectral determinant

$$
\left\langle\psi_{m}|A| \psi_{m}\right\rangle=-\left.\frac{\frac{\partial}{\partial \lambda} \Delta(E, \lambda)}{\frac{\partial}{\partial E} \Delta(E, \lambda)}\right|_{\substack{\lambda=0 \\ E=E_{m}}}
$$

The corresponding semiclassical approximation is given by

$$
\left\langle\psi_{m}|A| \psi_{m}\right\rangle \approx-\left.\frac{\operatorname{Re}\left[\frac{\partial}{\partial \lambda} \Delta(E, \lambda)\right]^{\mathrm{sc}, \mathrm{tr}}}{\operatorname{Re}\left[\frac{\partial}{\partial E} \Delta(E, \lambda)\right]^{\mathrm{sc}, \mathrm{tr}}}\right|_{\substack{\lambda=0 \\ E=E_{m}}}
$$

We insert the semiclassical approximation of the determinant (2) and perform the derivatives. This results in

$$
\left\langle\psi_{m}|A| \psi_{m}\right\rangle \approx \frac{\pi \bar{d}_{A}\left(E_{m}\right) \operatorname{Im}\left[\Delta\left(E_{m}\right)\right]^{\mathrm{sc}, \operatorname{tr}}-\frac{1}{\hbar} B\left(E_{m}\right) \operatorname{Im}\left[\mathrm{e}^{-\mathrm{i} \pi \bar{N}\left(E_{m}\right)} \sum_{n=1}^{\infty} A_{n} C_{n} \exp \left(\frac{\mathrm{i}}{\hbar} \mathcal{S}_{n}\right)\right]^{\operatorname{tr}}}{\pi \bar{d}\left(E_{m}\right) \operatorname{Im}\left[\Delta\left(E_{m}\right)\right]^{\mathrm{sc}, \operatorname{tr}}-\frac{1}{\hbar} B\left(E_{m}\right) \operatorname{Im}\left[\mathrm{e}^{-\mathrm{i} \pi \bar{N}\left(E_{m}\right)} \sum_{n=1}^{\infty} T_{n} C_{n} \exp \left(\frac{\mathrm{i}}{\hbar} \mathcal{S}_{n}\right)\right]^{\operatorname{tr}}} .
$$

Here

$$
\bar{d}_{A}(E)=\left.\frac{\partial}{\partial \lambda} \bar{N}(E)\right|_{\lambda=0} \sim-(2 \pi \hbar)^{-D} \int \mathrm{d}^{D} q \mathrm{~d}^{D} p A(\boldsymbol{q}, \boldsymbol{p}) \delta\left(E-H_{0}(\boldsymbol{q}, \boldsymbol{p})\right),
$$

where $A(\boldsymbol{q}, \boldsymbol{p})$ is the classical function corresponding to the operator $A$. Furthermore

$$
A_{n}=\sum_{p \in \mathcal{P}_{n}} m_{p} \frac{\partial S_{p}}{\partial \lambda}=-\sum_{p \in \mathcal{P}_{n}} m_{p} \int_{0}^{T_{p}} A(\boldsymbol{q}(t), \boldsymbol{p}(t)) \mathrm{d} t
$$

where the integral is evaluated along periodic orbits. The last equality can be obtained from [38, 39].

Note that both, $\bar{d}_{A}(E)$ and $\bar{d}(E)$, are proportional to $\hbar^{-D}$. If we take only these terms into account we obtain the following approximation

$$
\left\langle\psi_{m}|A| \psi_{m}\right\rangle \approx \frac{\bar{d}_{A}\left(E_{m}\right)}{\bar{d}\left(E_{m}\right)}
$$


This is the average of $A(\boldsymbol{q}, \boldsymbol{p})$ over the energy shell, in accordance with quantum ergodicity.

The authors of [40] express expectation values as a quotient of derivatives of the zeta function (4) which is modified by including additional terms in the Euler product. For the composite orbits these additional terms are factors of the form $\mathrm{e}^{-\lambda A_{n}}$. The resulting approximation differs from (49) in that zero-length terms were not included, and it does not involve a resummation. Instead a curvature expansion was applied. In [33] the scattering approach to quantization was applied to obtain expectation values of smooth operators in terms of Poincaré section wavefunctions.

\section{Discussion}

We have derived in this article semiclassical approximations for wavefunctions, Green's functions and expectation values in terms of a finite number of classical trajectories by relating them to spectral determinants. The main results are equations (35), (40), (42), and (48). These formulas have a simple and transparent form, and this might facilitate a theoretical study of semiclassical properties, for example scars, as well as a numerical evaluation. We have concentrated on the position representation, but it is not difficult to obtain other representations as well as is shown by the example of phase space representations in equations (44) and (45). Alternatively, one may apply different perturbations from the start.

One quantity that appears in almost all approximations as an additional weight of a trajectory $\gamma$ is the semiclassical spectral determinant which is smoothly truncated at half the Heisenberg time minus the time along the trajectory, $T_{H} / 2-T_{\gamma}$. Hence it would be of interest to understand the dependence of the truncated semiclassical spectral determinant on the cut-off. For systems with zero Maslov index one might investigate this dependence by using a Guinand-type approach, which involves approximating it by an integral [41].

As is demonstrated by the example in appendix $\mathrm{B}$ the method can also be applied to onedimensional systems. An open question is how the approach of this article is applicable to integrable systems with dimension $D>1$, or mixed systems. The relations between wavefunctions, etc., and spectral determinants in equations (34), (39), (41), and (47) are general and do not depend on the nature of the classical dynamics. What is needed is an explicit semiclassical expansion of the spectral determinant in terms of composite orbits for these systems, or a semiclassical approximation of spectral determinants that is based on the EBK-quantization for integrable systems.

\section{Acknowledgements}

I would like to thank Alfredo Ozorio de Almeida and Raul Vallejos and other members of the Centro Brasileiro de Pesquisas Físicas for their kind hospitality during a sabbatical leave. Helpful discussions with Jon Keating, Jens Marklof, Alfredo Ozorio de Almeida and Jonathan Robbins are gratefully acknowledged.

\section{A Regularization of spectral determinants}

This section discusses Weierstrass and zeta regularized spectral determinants and is based on [42]. It considers spectral sequences $\left\{E_{k}\right\}$ with $0<E_{0} \leq E_{1} \leq E_{2} \leq \ldots$ and $E_{k} \rightarrow \infty$ as 
$k \rightarrow \infty$. Further assumptions concern the partition function, or the trace of the heat kernel. It is assumed that its definition in terms of a sum over energies is convergent for $\operatorname{Re} t>0$, and that it has the following asymptotic expansion

$$
\Theta(t):=\sum_{k=0}^{\infty} \mathrm{e}^{-t E_{k}} \sim \sum_{n=0}^{\infty} c_{i_{n}} t^{i_{n}}, \quad \text { as } \quad t \rightarrow 0
$$

with an increasing sequence of real $i_{n}$ without limit point and $i_{0}<0$. Let $\mu=-i_{0}$ in the following. Equation (53) implies that the leading term in Weyl's law for the spectral counting function is given by

$$
N(E) \sim \frac{c_{i_{0}}}{\Gamma\left(1-i_{0}\right)} E^{\mu}
$$

There are different ways to define a spectral determinant by a convergent product over the eigenvalues $E_{k}$ of the form (1). The Weierstrass canonical product is defined by

$$
\Delta_{W}(E)=\prod_{k=0}^{\infty}\left(1-\frac{E}{E_{k}}\right) \exp \left(\frac{E}{E_{k}}+\frac{E^{2}}{2 E_{k}^{2}}+\cdots+\frac{E^{[\mu]}}{[\mu] E_{k}^{[\mu]}}\right),
$$

where $[\mu]$ is the integer part of $\mu$. An alternative regularization, the zeta regularization, defines the spectral determinant by an analytic continuation of the generalized zeta function

$$
Z(s, a)=\sum_{k=0}^{\infty}\left(E_{k}+a\right)^{-s}=\Gamma(s)^{-1} \int_{0}^{\infty} \Theta(t) \mathrm{e}^{-a t} t^{s-1} \mathrm{~d} t .
$$

This definition is valid for $\operatorname{Re} s>\mu$ and $a>-E_{0}$ and is extended elsewhere by analytic continuation. The determinant is defined as

$$
\Delta_{Z}(E)=\exp \left[-Z^{\prime}(0,-E)\right]
$$

where the derivative is with respect to the first variable. Both determinants, $\Delta_{W}(E)$ and $\Delta_{Z}(E)$, are entire functions of $E$. The relation between both determinants can be understood by looking at the trace of the $\mu$-th derivative of the resolvent which is given by the convergent sum

$$
\operatorname{Tr} G^{([\mu])}(E)=(-1)^{[\mu]}[\mu] ! \sum_{k=0}^{\infty}\left(E-E_{k}\right)^{-[\mu]-1} .
$$

(Note that the resolvent is defined with a different sign in [42]). It can be shown that both determinants can be obtained by a multiple integration of (58). The difference is that the lower integration limit is $E=0$ for the Weierstrass regularized determinant whereas it is $E=-\infty$ for the zeta regularized determinant

$$
\log \Delta_{W}(E)=\left(\int_{0}^{E}\right)^{[\mu+1]} \operatorname{Tr} G^{([\mu])}(E), \quad \log \Delta_{Z}(E)=\left(\int_{-\infty}^{E}\right)^{[\mu+1]} \operatorname{Tr} G^{([\mu])}(E) .
$$

In the second case the integral is defined by finite part extractions when necessary. For example, the following rules hold

$$
\begin{aligned}
& \int_{-\infty}^{E}\left(-E^{\prime}\right)^{-s} \mathrm{~d} E^{\prime}=\frac{(-E)^{1-s}}{s-1}, \quad(s \neq 1), \quad \int_{-\infty}^{E}\left(-E^{\prime}\right)^{-1} \mathrm{~d} E^{\prime}=-\log (-E), \\
& \int_{-\infty}^{E} \log \left(-E^{\prime}\right) \mathrm{d} E^{\prime}=E(\log (-E)-1) .
\end{aligned}
$$


It can be further shown that both determinants are related by

$$
\Delta_{Z}(E)=\Delta_{W}(E) \exp \left(-\sum_{m=0}^{[\mu]} a_{m} E^{m}\right)
$$

Expressions for the constants $a_{m}$ can be found in [42].

Semiclassical approximations for the spectral determinants are obtained by starting with the semiclassical expression for the trace of the $[\mu]$-derivative of the resolvent and doing the integrations in (59). The zeta regularized determinant is convenient for semiclassical approximations, because the periodic orbit terms do not give a contribution at $E=-\infty$. If the periodic orbit terms are integrated for chaotic systems with unstable periodic orbits they yield the dynamical zeta function $\zeta(E)$ in (41) [19, 3]. The asymptotics of $\operatorname{Tr} G^{([\mu]}(E)$ in the limit $E \rightarrow-\infty$ is determined by the zero-length contributions. From the asymptotic expansion of the trace of the heat kernel (53) follows that

$$
\operatorname{Tr} G^{([\mu])}(E)=-\Gamma([\mu]+1) Z([\mu]+1,-E) \sim-\sum_{n=0}^{\infty} c_{i_{n}} \Gamma\left([\mu]+1+i_{n}\right)(-E)^{-i_{n}-[\mu]-1},
$$

as $E \rightarrow-\infty$. For the example of a two-dimensional billiard system with Dirichlet boundary conditions (units $\hbar=2 m=1$ ) the first three coefficients are $c_{-1}=A /(4 \pi), c_{-1 / 2}=-L /(8 \sqrt{\pi})$, $c_{0}=C$, and $\mu=1$. Here $A$ is the area of the billiard, $L$ is its perimeter and $C$ is determined by the curvature and corners of the boundary [43]. The integration of (62) with the rules (60) then yields the zero-length contributions to the spectral determinant. We state here the result for two-dimensional billiard systems

$$
\Delta_{Z}(E) \approx \exp \{A E(\log (E)-1) / 4 \pi+C \log (E)-i \pi \bar{N}(E)\} \zeta(E),
$$

where

$$
\bar{N}(E) \approx \frac{A}{4 \pi} E-\frac{L}{4 \pi} \sqrt{E}+C .
$$

Equation (63) is in agreement with the appendix of [7]. Comparison with (2) shows that in the approximaton (63) for the zeta regularized determinant the function $B(E)$ is given by

$$
B_{Z}(E)=\exp \{A E(\log (E)-1) / 4 \pi+C \log (E)\}
$$

It is part of the zero-length contribution. For the Weierstrass regularization $B(E)$ contains an additional factor according to (61).

\section{B A one-dimensional example}

In this section we apply the resummation method to a one-dimensional example. In order to test the formula for complex-valued Green's functions we consider a system without timereversal symmetry. It will be seen that the resummation is exact if we choose a sharp cut-off by a Heaviside theta function.

$$
\theta^{\operatorname{tr}}(T)=\theta(T)=\left\{\begin{array}{lll}
0 & \text { if } & T<0 \\
\frac{1}{2} & \text { if } & T=0 \\
1 & \text { if } & T>0
\end{array}\right.
$$


The Schrödinger equation is given in units where $\hbar=2 m=1$ by

$$
\left(-i \frac{\mathrm{d}}{\mathrm{d} q}+\alpha\right)^{2} \psi(q)=E \psi(q)
$$

where $\alpha$ is constant, and we apply periodic boundary conditions of the form $\psi(q+l)=\psi(q)$. The solutions for (67) are given by

$$
\psi_{n}(q)=\frac{1}{\sqrt{l}} \mathrm{e}^{\mathrm{i} 2 \pi n q / l}, \quad E_{n}=\left(\frac{2 \pi n}{l}+\alpha\right)^{2}, \quad n \in \mathbb{Z} .
$$

The corresponding free Green's function is obtained from its differential equation $\left(E=k^{2}\right)$

$$
G^{\text {free }}\left(q, q^{\prime}, E\right)=\frac{1}{2 \mathrm{i} k} \mathrm{e}^{\mathrm{i} k\left|q-q^{\prime}\right|-\mathrm{i} \alpha\left(q-q^{\prime}\right)} .
$$

The Green's function of the considered system follows by the method of images

$$
G_{0}\left(q, q^{\prime}, E\right)=\sum_{n=-\infty}^{\infty} G^{\mathrm{free}}\left(q+n l, q^{\prime}, E\right)
$$

This representation of the Green's function is identical to its semiclassical approximation. The sum can be interpreted as sum over all trajectories from $q^{\prime}$ to $q$ with lengths $L_{n}=\left|q+n l-q^{\prime}\right|$. The sum in (70) involves only geometric series and can be carried out with the result

$$
G_{0}\left(q, q^{\prime}, E\right)=\frac{\mathrm{e}^{-\mathrm{i} \alpha\left(q-q^{\prime}\right)}\left[\sin (\alpha l) \mathrm{e}^{\mathrm{i} k\left(q-q^{\prime}\right)}+\sin \left(k l-k\left|q-q^{\prime}\right|\right)-\sin \left(\alpha l-k\left|q-q^{\prime}\right|\right)\right]}{2 k(\cos (\alpha l)-\cos (k l))} .
$$

For $q^{\prime}=q$ we obtain

$$
G_{0}(q, q, E)=\frac{1}{2 \mathrm{i} k}+\sum_{n=1}^{\infty} \frac{1}{\mathrm{i} k} \mathrm{e}^{\mathrm{i} k n l} \cos (\alpha n l)=\frac{\sin (k l)}{2 k(\cos (\alpha l)-\cos (k l))} .
$$

From the zero-length term we obtain the mean spectral staircase $\bar{N}(E)=k l / \pi$. Instead of the Heisenberg time it is more convenient to speak of the Heisenberg length which is $L_{H}=2 k T_{H}=$ $4 k \pi \bar{N}^{\prime}(E)=2 l$. Hence the cut-off for the orbit sums is at $l$.

The spectral determinant is

$$
\Delta_{0}(E)=\exp \left(\int \mathrm{d} E \operatorname{Tr} G_{0}(E)\right)=\cos (\alpha l)-\cos (k l) .
$$

This can be written in the form

$$
\Delta_{0}(E)=\mathrm{e}^{-\mathrm{i} k l}\left[-\frac{1}{2}+\cos (\alpha l) \mathrm{e}^{\mathrm{i} k l}-\frac{1}{2} \mathrm{e}^{2 \mathrm{i} k l}\right],
$$

which corresponds to the sum over composite orbits in this one-dimensional system. Applying the resummation with the theta-function as cut-off we find that

$$
\Delta_{0}(E) \approx 2 \operatorname{Re}\left[\Delta_{0}(E)\right]^{\mathrm{sc}, \operatorname{tr}}=2 \operatorname{Re} \mathrm{e}^{-\mathrm{i} k l}\left[-\frac{1}{2}+\frac{1}{2} \cos (\alpha l) \mathrm{e}^{\mathrm{i} k l}\right]=\cos (\alpha l)-\cos (k l) .
$$


Hence the resummation is exact.

Let us apply now the resummation to the Green's function

$$
G_{0}\left(q, q^{\prime}, E\right) \approx \frac{\left[\Delta_{0}(E) G_{0}\left(q, q^{\prime}, E\right)+\overline{\Delta_{0}(E) G_{0}\left(q^{\prime}, q, E\right)}\right]^{\mathrm{sc}, \mathrm{tr}}}{2 \operatorname{Re}\left[\Delta_{0}(E)\right]^{\mathrm{sc}, \mathrm{tr}}} .
$$

One has to multiply the sums (70) and (174) and discard all terms for which the joint lengths of the trajectories is larger than $L_{H} / 2=l$ (and multiply terms for which the joint lengths is $l$ by $1 / 2$ ). We consider first the case $q^{\prime}=q$ and obtain

$$
\begin{aligned}
G_{0}(q, q, E) & =2 \operatorname{Re} \frac{\frac{1}{2 \mathrm{i} k} \mathrm{e}^{-\mathrm{i} k l}\left[-\frac{1}{2}+\frac{1}{2} \cos (\alpha l) \mathrm{e}^{\mathrm{i} k l}-\frac{1}{4} \mathrm{e}^{\mathrm{i} k l-\mathrm{i} \alpha l}-\frac{1}{4} \mathrm{e}^{\mathrm{i} k l+\mathrm{i} \alpha l}\right]}{\cos (\alpha l)-\cos (k l)} \\
& =\frac{\sin (k l)}{2 k[\cos (\alpha l)-\cos (k l)]},
\end{aligned}
$$

which agrees with (72). In the case $q^{\prime} \neq q$

$$
\begin{aligned}
G_{0}\left(q, q^{\prime}, E\right) & =\frac{\frac{1}{4 i k} \mathrm{e}^{-\mathrm{i} k l}\left[-\mathrm{e}^{\mathrm{i} k\left|q-q^{\prime}\right|-\mathrm{i} \alpha\left(q-q^{\prime}\right)}-\mathrm{e}^{\mathrm{i} k\left(l-\left|q-q^{\prime}\right|\right)-\mathrm{i} \alpha\left(q-q^{\prime} \mp l\right)}\right]}{\cos (\alpha l)-\cos (k l)} \\
& +\frac{-\frac{1}{4 \mathrm{i} k} \mathrm{e}^{\mathrm{i} k l}\left[-\mathrm{e}^{-\mathrm{i} k\left|q-q^{\prime}\right|-\mathrm{i} \alpha\left(q-q^{\prime}\right)}-\mathrm{e}^{-\mathrm{i} k\left(l-\left|q-q^{\prime}\right|\right)-\mathrm{i} \alpha\left(q-q^{\prime} \mp l\right)}\right]}{\cos (\alpha l)-\cos (k l)} \\
& =\frac{\sin \left(k l-k\left|q-q^{\prime}\right|\right)+\sin \left(k\left|q-q^{\prime}\right|\right) \mathrm{e}^{ \pm \mathrm{i} \alpha l}}{2 k[\cos (\alpha l)-\cos (k l)]} \mathrm{e}^{-\mathrm{i} \alpha\left(q-q^{\prime}\right)},
\end{aligned}
$$

where the upper and lower signs in the exponents correspond to the cases $q>q^{\prime}$ and $q<q^{\prime}$, respectively. A short calculation shows that (178) is identical to (71).

\section{References}

[1] M. C. Gutzwiller: Chaos in Classical and Quantum Mechanics, Springer, New York, (1990).

[2] H.-J. Stöckmann: Quantum Chaos, Cambridge University Press, Cambridge, (1999).

[3] M. V. Berry and J. P. Keating, J. Phys. A 23 (1990) 4839-4849.

[4] M. V. Berry and J. P. Keating, Proc. R. Soc. Lond. A 437 (1992) 151-173.

[5] E. B. Bogomolny, Nonlinearity 5 (1992) 805-866.

[6] E. Doron and U. Smilansky, Nonlinearity 5 (1992) 1055-1084.

[7] J. P. Keating and M. Sieber, Proc. R. Soc. Lond. A 447 (1994) 413-437.

[8] E. B. Bogomolny, Physica D 31 (1988) 169-189.

[9] M. V. Berry, Proc. R. Soc. Lond. A 423 (1989) 219-231.

[10] E. Heller, Phys. Rev. Lett. 53 (1984) 1515-1518.

[11] O. Agam and S. Fishman, J. Phys. A 26 (1993) 2113-2137, Corrigendum in J. Phys. A 26 (1993) 6595 . 
[12] O. Agam and S. Fishman, Phys. Rev. Lett. 73 (1994) 806-809.

[13] B. Georgeot and R. E. Prange, Phys. Rev. Lett. 74 (1995) 2851-2854.

[14] S. Fishman, B. Georgeot and R. E. Prange, J. Phys. A 29 (1996) 919-937.

[15] R. E. Prange, J. Stat. Phys. 93 (1998) 965-980.

[16] F. P. Simonotti and M. Saraceno, Phys. Rev. E 61 (2000) 6527-6537.

[17] M. Saraceno and A. G. Monastra, nlin.CD/0105006, unpublished.

[18] O. M. Auslaender and S. Fishman, Physica D 128 (1999) 180-223.

[19] A. Voros, J. Phys. A 21 (1988) 685-692.

[20] J. P. Keating, Proc. R. Soc. Lond. 436 (1992) 99-108.

[21] M. Gutzwiller, J. Math. Phys. 8 (1967) 1979-2000.

[22] M. V. Berry and K. E. Mount, Rep. Prog. Phys. 35 (1972) 315-397.

[23] M. Abramowitz and I. A. Stegun: Handbook of Mathematical Functions, National Bureau of Standards, Washington, D.C., (1964).

[24] G. Vattay, A. Wirzba and P. E. Rosenqvist, Phys. Rev. Lett. 73 (1994) 2304-2307.

[25] S. Albeverio, F. Gesztesy, R. Høegh-Krohn and H. Holden: Solvable Models in Quantum Mechanics, Springer, New York, (1988).

[26] S. Albeverio and P. Kurasov: Singular Perturbations of Differential Operators, London Mathematical Society Lecture Notes 271, Cambridge University Press, Cambridge, (2000).

[27] J. Zorbas, J. Math. Phys. 21 (1980) 840-847.

[28] R. Jackiw, in: M. A. B. Bég Memorial Volume (Eds. A. Ali and P. Hoodbhoy), 25-42. World Scientific, Singapore, (1991).

[29] P. Kurasov, in: Spectral Theory of Schrödinger Operators, Contemporary Mathematics 340 (Eds. R. del Rio and C. Villegas-Blas), 185-216. Americal Mathematical Society, (2004).

[30] P. Exner and P. Šeba, Phys. Lett. A 222 (1996) 1-4.

[31] L. Dabrowski and H. Grosse, J. Math. Phys. 26 (1985) 2777-2780.

[32] S. Albeverio and P. Kurasov, Proc. Amer. Math. Soc. 127 (1999) 1151-1161.

[33] D. Klakow and U. Smilansky, J. Phys. A 29 (1996) 3213-3231.

[34] A. I. Shnirelman, Usp. Math. Nauk 29 (1974) 181-182.

[35] Y. Colin de Verdière, Commun. Math. Phys. 102 (1985) 111-134.

[36] S. Zelditch, Duke Math. J. 55 (1987) 919-941.

[37] A. M. Ozorio de Almeida, Phys. Rep. 295 (1998) 265-344.

[38] J. Goldberg, U. Smilansky, M. V. Berry, W. Schweizer, G. Wunner and G. Zeller, Nonlinearity 4 (1991) 1-14. 
[39] A. M. Ozorio de Almeida, C. H. Lewenkopf and E. R. Mucciolo, Phys. Rev. E 58 (1998) 56935703.

[40] B. Eckhardt, S. Fishman, K. Müller and D. Wintgen, Phys. Rev. A 45 (1992) 3531-3539.

[41] M. Berry, in: Les Houches 1989 Session LII on Chaos and Quantum Physics (Eds. M. J. Giannoni, A. Voros and J. Zinn-Justin), 251-303. North-Holland, Amsterdam, (1991).

[42] A. Voros, Commun. Math. Phys. 110 (1987) 439-465.

[43] H. P. Baltes and E. R. Hilf: Spectra of Finite Systems, B.I.-Wissenschaftsverlag, Mannheim, (1976). 